\title{
Sustained High D-Dimer in Outpatients Who Have Recovered from Mild to Moderate Coronavirus Disease 2019 (COVID-19)
}

\author{
Serafino Fazio, $\mathrm{MD}^{1}$ Antonella Tufano, MD, $\mathrm{PhD}^{2}$ \\ ${ }^{1}$ Department of Advanced Biomedical Sciences, Federico II \\ University, Naples, Italy \\ 2 Department of Clinical Medicine and Surgery, Federico II University, \\ Naples, Italy
}

Semin Thromb Hemost 2022;48:115-117.

\author{
Giovanni de Simone, MD ${ }^{1}$
}

Over 1 year has passed since the beginning of the coronavirus disease 2019(COVID-19) pandemic outbreak. Throughout this period of time, severe acute respiratory syndrome coronavirus-2 (SARS-CoV2) has spread across the globe, causing a disease that generates high and fast rates of hospitalizations and deaths. It has been now well clarified that COVID-19 is not just a respiratory disease, but rather in the most severe cases is the result of inappropriate response of immune system precipitating a so-called "cytokine storm" and immunothrombosis, accompanied by vasculitis, micro- and macro-angiopathy, thromboembolism, and multiple organ damage, especially if prompt treatment is not undertaken. ${ }^{1}$

Very recently, the American Society of Hematology (ASH) released new guidelines for anticoagulation in COVID-19 patients. ${ }^{2}$ In the context of "very low certainty of evidence," as stated, ASH confirmed recommendation for using prophylactic-intensity rather than intermediate-intensity or therapeutic-intensity anticoagulation in critical COVID-19 patients, without confirmed or suspected venous thromboembolism, consistent with some evidence that in critically ill patients, high dose anticoagulation does not exhibit the same benefit, and rather, can increase mortality. ${ }^{3}$

Several studies report high incidence of vascular thrombosis (arterial and venous thromboembolism, pulmonary and cardiac microthrombi, disseminated intravascular coagulation [DIC]) in patients with COVID-19. Elevated levels of some markers of coagulation activation are among the frequent findings in the most severe patients admitted to the intensive care unit (ICU). Most recently, the pathology of COVID-19-related coagulopathy has been unraveled. ${ }^{4,5} \mathrm{~A}$ necropsy study revealed that $64 \%$ of cases of myocardial necrosis was due to microthrombi, related not directly to cardiac viral invasion, with distinct high levels of fibrin and terminal complement, different from thrombi found in epicardial coronary arteries during acute myocardial infarc-

published online May 7, 2021
Issue Theme Maintaining Hemostasis and Preventing Thrombosis in COVID-19-Part III; Guest Editors: Emmanuel J. Favaloro, PhD, FFSc (RCPA) and Giuseppi Lippi, MD

tion. ${ }^{4}$ Elevated D-dimer is likely the most appropriate marker of this type of fibrin-related micro-thrombosis, as the direct expression of plasmin activity on stabilized fibrin crosslinks. The evidence of fibrin clots in the myocardial microcirculation and the high incidence of venous and arterial thromboembolic events in COVID-19 patients raise questions about the use of prophylactic, intermediate, or therapeutic-intensity low-molecular weight heparin (LMWH) in COVID-19 patients, independently of the overt clinical presentation of thromboembolism, prompting NHLBI to issue a public notice on preliminary results from three clinical trials showing clear benefit of high-dose anticoagulation over prophylactic intensity in moderately ill patients. ${ }^{6}$

The apparently contradictory current indications are actually consistent with the importance of using the right therapeutic decision according to the stage of the disease. ${ }^{7}$ Heparin treatment may be of critical value during the formation of microthrombi, to prevent them and, therefore, before stage 3 (hyperinflammation) and probably even before stage 2 , when the disease shifts from the phase of viral invasion toward the phase of hyperimmune response. ${ }^{8}$ In addition, the use of prophylactic doses of LMWH or unfractionated heparin reduces the 28-day mortality in very sick COVID-19 patients (in the presence of D-dimer levels more than sixfold of the upper limit of normal or a Sepsis-Induced Coagulopathy score $\geq 4) .{ }^{9}$ The thromboembolic risk extends for up to 6 weeks post-hospital discharge in high-risk medically ill patients, including patients with pneumonia and sepsis. A modified IMPROVE (International Medical Prevention Registry on Venous Thromboembolism) risk score, using established cut-offs plus elevated D-dimer ( $>2$ times upper normal limit [UNL]) identifies patients at an almost threefold higher thromboembolic risk. ${ }^{10}$ In these patients, significant benefits are reported when extending thromboprophylaxis. ${ }^{10}$

(C) 2021. Thieme. All rights reserved. Thieme Medical Publishers, Inc., 333 Seventh Avenue, 18th Floor, New York, NY 10001, USA
DOI https://doi.org/ 10.1055/s-0041-1729857. ISSN 0094-6176. 
Based on this evidence, extended post-discharge thromboprophylaxis should be considered for all hospitalized patients with COVID-19 who meet high VTE risk criteria. The duration of post-discharge thromboprophylaxis is suggested to be at least 14 days. ${ }^{11}$ Among the many laboratorymeasurable prothrombotic biomarkers, D-dimer seems particularly effective in identifying impending thromboembolic activity. ${ }^{12}$ Elevated D-dimer levels at admission or increasing D-dimer over time are both associated with increased mortality with COVID-19. ${ }^{5}$

Rising D-dimer after admission precedes multiple organ failure and overt DIC. Moreover, longer duration of hospital stay is associated with increasing D-dimer. ${ }^{13}$ However, in the context of noncritical COVID-19, the value of monitoring Ddimer also in out-of-hospital mild/moderate disease remains unclear, ${ }^{14}$ a question requiring promptanswer, given the efforts to identify predictors of severe disease at the early stages. ${ }^{15}$

We herein report evidence of persisting high D-dimer levels in $20 \%$ of the patients with mild to moderate COVID-19, who requested remote consultation and were, therefore, remotely followed at home. This is a series of 45 patients with mild to moderate COVID-19 (age $51 \pm 14$ years, 21 women).

Diagnosis of COVID-19 was confirmed in out-of-hospital private laboratories authorized to perform molecular tests, by nasal swab for nucleic acid amplification test to detect SARS-Cov-2 mRNA, using different techniques, mostly by the reverse transcription polymerase chain reaction (RT-PCR). Ddimer was measured using different immunoassays, giving different normal limits. Given the impossibility to directly compare D-dimer values produced with different methods, ${ }^{16}$ the D-dimer value was expressed as the percent deviation from the upper normal limit (UNL) that every laboratory gave in their reports.

COVID-19 patients came to our observation at different times from beginning of symptoms. All patients were treated with indomethacin 75 to $150 \mathrm{mg} / \mathrm{d}$ according to the patient weight, omeprazole $20 \mathrm{mg} / \mathrm{d}$, and enoxaparin $4000 \mathrm{IU} / \mathrm{d}$. In 10 patients, in whom initial symptoms and signs of COVID19 (anosmia/ageusia, sore throat, fever, fatigue, muscular pain, cold, dry cough, diarrhea, abdominal pain, shortness of breath in different combinations) persisted over 6 days (two of them in the subgroup with persistent high D-dimer), a 6day course of prednisone $25 \mathrm{mg} /$ bid was added.

After complete clinical recovery, restoration of a wellbeing and normalization of molecular swab, C-reactive protein, white blood cell count and fibrinogen level, D-dimer remained substantially elevated in nine patients $(20 \%)$, with high levels persisting at 15 days after complete clinical and laboratory remission ( - Fig. $\mathbf{1}$; note that the reported values are the percent increase referenced against the UNL specific for each laboratory). Patients with persistently high D-dimer had the same sex distribution (four women) as the subgroup with normal $D$-dimer but were slightly younger ( $47 \pm 13$ vs. $51 \pm 13$ years, $p=0.05$ ).

Our study has obvious limitations, including that D-dimer was measured with a variety of methods, and thus not strictly comparable. ${ }^{16}$ However, we believe that reporting

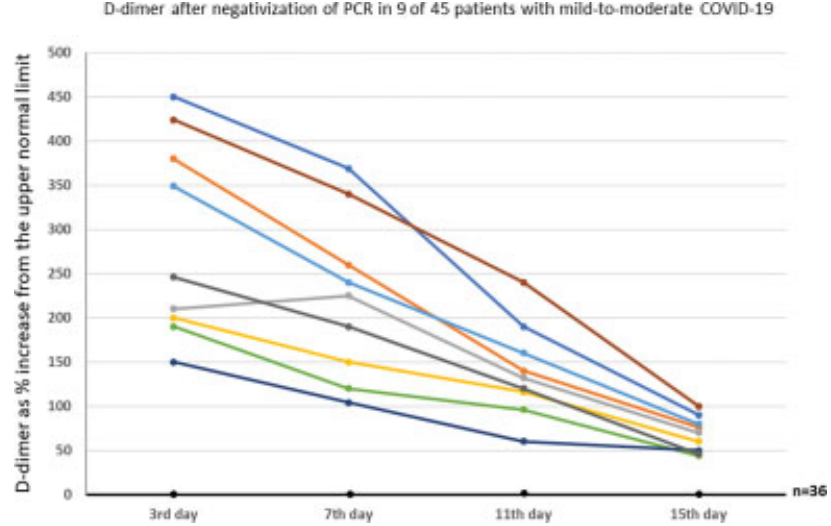

Fig. 1 D-dimer monitoring over 15 days, in 9 of 45 patients maintaining high values after clinical recovery and negativization of swab.

data as a percent increase over the UNL represent a reasonable approach. Also, our empiric, clinical observation needs confirmation. However, the data suggests that D-dimer should be monitored also in mild-to-moderate COVID-19 patients, even if managed at home. In addition, our experience also raises several questions:

1. Should anticoagulation (e.g., LMWH) be administered in all mild-to-moderate COVID-19 patients or at least in those who exhibit elevated D-dimer (implying that in all patients D-dimer should be measured), and for how long?

2. Is prophylactic-intensity LMWH sufficient to prevent microthrombus formation that is clinically silent?

3. Finally, can persistence of elevated D-dimer beyond the clinical recovery be a track to understand mechanisms leading to the frequent long-term consequences of SARSCoV-2 infection? ${ }^{17}$

All these questions need attention and well-designed observational studies.

Conflict of Interest

None declared.

\section{References}

1 Bikdeli B, Madhavan MV, Jimenez D, et al; Global COVID-19 Thrombosis Collaborative Group, Endorsed by the ISTH, NATF, ESVM, and the IUA, Supported by the ESC Working Group on Pulmonary Circulation and Right Ventricular Function. COVID-19 and thrombotic or thromboembolic disease: implications for prevention, antithrombotic therapy, and follow-up: JACC State-of-the-Art Review. J Am Coll Cardiol 2020;75(23): 2950-2973

2 Cuker A, Tseng EK, Nieuwlaat R, et al. American Society of Hematology 2021 guidelines on the use of anticoagulation for thromboprophylaxis in patients with COVID-19. Blood Adv 2021; 5(03):872-888

3 Lynn L, Reyes JA, Hawkins K, et al. The effect of anticoagulation on clinical outcomes in novel Coronavirus (COVID-19) pneumonia in a U.S. cohort. Thromb Res 2021;197:65-68

4 Pellegrini D, Kawakami R, Guagliumi G, et al. Microthrombi as a major cause of cardiac injury in COVID-19: a pathologic study. Circulation 2021;143(10):1031-1042 
5 Hadid T, Kafri Z, Al-Katib A. Coagulation and anticoagulation in COVID-19. Blood Rev 2021;47:100761

6 NHLBI NEWS. Full-dose blood thinners decreased need for life support and improved outcome in hospitalized COVID-19 patients. Accessed January 22, 2021 at: https://www.nih.gov/news-events/news-releases/full-dose-blood-thinners-decreased-need-life-support-improved-outcome-hospitalizedcovid-19-patients

7 de Simone G, Mancusi C. CoVID-19: timing is important. Eur J Intern Med 2020;77:134-135

8 Siddiqi HK, Mehra MR. COVID-19 illness in native and immunosuppressed states: a clinical-therapeutic staging proposal. J Heart Lung Transplant 2020;39(05):405-407

9 Tang N, Bai H, Chen X, Gong J, Li D, Sun Z. Anticoagulant treatment is associated with decreased mortality in severe coronavirus disease 2019 patients with coagulopathy. J Thromb Haemost 2020;18(05):1094-1099

10 Spyropoulos AC, Lipardi C, Xu J, et al. Modified IMPROVE VTE risk score and elevated D-dimer identify a high venous thromboembolism risk in acutely ill medical population for extended thromboprophylaxis. TH Open 2020;4(01): e59-e65
11 Spyropoulos AC, Levy JH, Ageno W, et al; Subcommittee on Perioperative, Critical Care Thrombosis, Haemostasis of the Scientific, Standardization Committee of the International Society on Thrombosis and Haemostasis. Scientific and Standardization Committee communication: Clinical guidance on the diagnosis, prevention, and treatment of venous thromboembolism in hospitalized patients with COVID-19. J Thromb Haemost 2020;18(08):1859-1865

12 Hardy $M$, Lecompte $T$, Douxfils J, et al. Management of the thrombotic risk associated with COVID-19: guidance for the hemostasis laboratory. Thromb J 2020;18:17

13 Connors JM, Levy JH. COVID-19 and its implications for thrombosis and anticoagulation. Blood 2020;135(23):2033-2040

14 Emert R, Shah P, Zampella JG. COVID-19 and hypercoagulability in the outpatient setting. Thromb Res 2020;192:122-123

15 Li X, Marmar T, Xu Q, et al. Predictive indicators of severe COVID19 independent of comorbidities and advanced age: a nested case-control study. Epidemiol Infect 2020;148:e255

16 Favaloro EJ, Thachil J. Reporting of D-dimer data in COVID-19: some confusion and potential for misinformation. Clin Chem Lab Med 2020;58(08):1191-1199

17 Del Rio C, Collins LF, Malani P. Long-term health consequences of COVID-19. JAMA 2020;324(17):1723-1724 